\title{
High-Performance Modeling of Carbon Dioxide Sequestration by Coupling Reservoir Simulation and Molecular Dynamics
}

\author{
Kai Bao', King Abdullah University of Science and Technology; Mi Yan², IBM; Rebecca Allen ${ }^{3}$ and \\ Amgad Salama ${ }^{4}$, King Abdullah University of Science and Technology; Ligang Lu ${ }^{5}$ and Kirk E. Jordan, IBM; \\ Shuyu Sun and David Keyes, King Abdullah University of Science and Technology
}

\begin{abstract}
Summary
The present work describes a parallel computational framework for carbon dioxide $\left(\mathrm{CO}_{2}\right)$ sequestration simulation by coupling reservoir simulation and molecular dynamics (MD) on massively parallel high-performance-computing (HPC) systems. In this framework, a parallel reservoir simulator, reservoir-simulation toolbox (RST), solves the flow and transport equations that describe the subsurface flow behavior, whereas the MD simulations are performed to provide the required physical parameters. Technologies from several different fields are used to make this novel coupled system work efficiently.

One of the major applications of the framework is the modeling of large-scale $\mathrm{CO}_{2}$ sequestration for long-term storage in subsurface geological formations, such as depleted oil and gas reservoirs and deep saline aquifers, which has been proposed as one of the few attractive and practical solutions to reduce $\mathrm{CO}_{2}$ emissions and address the global-warming threat. Fine grids and accurate prediction of the properties of fluid mixtures under geological conditions are essential for accurate simulations. In this work, $\mathrm{CO}_{2}$ sequestration is presented as a first example for coupling reservoir simulation and $\mathrm{MD}$, although the framework can be extended naturally to the full multiphase multicomponent compositional flow simulation to handle more complicated physical processes in the future.

Accuracy and scalability analysis are performed on an IBM BlueGene/P and on an IBM BlueGene/Q, the latest IBM supercomputer. Results show good accuracy of our MD simulations compared with published data, and good scalability is observed with the massively parallel HPC systems. The performance and capacity of the proposed framework are well-demonstrated with several experiments with hundreds of millions to one billion cells.

To the best of our knowledge, the present work represents the first attempt to couple reservoir simulation and molecular simulation for large-scale modeling. Because of the complexity of subsurface systems, fluid thermodynamic properties over a broad range of temperature, pressure, and composition under different geological conditions are required, although the experimental results are limited. Although equations of state can reproduce the existing experimental data within certain ranges of conditions, their extrapolation out of the experimental data range is still limited. The present framework will definitely provide better flexibility and predictability compared with conventional methods.
\end{abstract}

\footnotetext{
${ }^{1}$ now with SINTEF ICT

${ }^{2}$ now with lonic Security

${ }^{3}$ now with SINTEF ICT

${ }^{4}$ now with Reservoir Engineering Research Institute

${ }^{5}$ now with Shell International Exploration and Production

Copyright $(C 2015$ Society of Petroleum Engineers
}

This paper (SPE 163621) was accepted for presentation at the SPE Reservoir Simulation Symposium, The Woodlands, Texas, USA, 8-20 February 2013, and revised for publication. Original manuscript received for review 25 August 2013. Revised manuscript received for review 19 May 2015. Paper peer approved 10 August 2015.

\section{Introduction}

$\mathrm{CO}_{2}$ sequestration for long-term storage in subsurface geological formulations, such as depleted oil and gas reservoirs and deep saline aquifers, has been proposed as one of the few feasible and practical solutions to reduce the $\mathrm{CO}_{2}$ emissions and address the global-warming threat (IPCC 2005; Firoozabadi and Cheng 2010; Michael et al. 2011). Because of the complexity of subsurface systems and the physical process involved, fine grids and accurate prediction of the fluid properties under subsurface conditions play an essential role. Under reservoir conditions, broad ranges of temperature, pressure, and fluid composition are possible, and the accurate prediction under certain geological conditions remains challenging.

Much experimental data have been accumulated and used to predict the fluid properties in reservoir simulation. However, compared with the large range of temperature and pressure under reservoir conditions, the experimental data are of limited applicability. Furthermore, as the temperature and pressure increase, experiments become more and more difficult and the accuracy of the measurement can be problematic. Various equations of state (EOSs) have also been proposed and extensively used for the prediction of the fluid properties under reservoir conditions. With EOSs, the existing experimental data with certain ranges of parameters can be accurately reproduced, although their extrapolation out of the experimental data range remains limited (Zhang and Duan 2005a).

Recently, molecular simulations-molecular dynamics (MD) and Monte Carlo- have become popular for predicting fluid properties under geological conditions (Zhang and Duan 2005a). Compared with the conventional experimental and EOS approaches, molecular simulations depend little on experimental conditions and have fewer limitations on ranges of temperature and pressure. Thus, molecular simulations hold the potential for better predictability in the study of fluid properties under geological conditions.

Also, different multiscale hybrid approaches have been developed to solve subsurface multiphysics problems during recent years, and most of the published works are focused on the coupling between pore scale and continuum scale. Tartakovsky et al. (2008) developed a coupled pore-continuum model with smoothed-particle hydrodynamics to handle reaction-diffusion processes in granular porous media. With the approach, the coupling of the two scales will be handled in a natural way on the internal boundaries between subdomains. In Balhoff et al. (2007), a pore-network model is coupled to a continuum model with a domain-decomposition approach. The flow rates entering/exiting the continuum match the flow exiting/entering the pore-network model through an iterative determination process of the boundary pressure. Battiatoa et al. (2011) treat the flux exchange between the two scales as unknowns and an iterative process is applied at the pore scale to guarantee the flux continuity. Mortars have been also used to handle multiscale problems (Balhoff et al. 2008; Sun et al. 2012). The heterogeneous multiscale method (E and Engquist 2003) is used to couple the continuum-scale mass-conservation law with a discrete network model for steady-state single-phase flow (Chu et al. 2012) and then extended to single-phase flow with nonlinear flux- 
pressure dependence and two-phase-flow problems (Chu et al. 2013). Sheng and Thompson (2013) provide a hybrid concurrent approach to couple a dynamic pore-network model to a continuum-scale simulator for multiphase-flow problems.

In this study, a parallel computational framework for $\mathrm{CO}_{2}$ sequestration simulation by coupling reservoir simulation and MD on massively parallel high-performance-computing systems is presented. In this framework, a parallel reservoir simulator, reservoir-simulation toolbox, is used to solve the flow and transport equations that describe the subsurface-flow behavior, whereas the MD simulations are performed to provide the required physical parameters. A coupler is developed to efficiently mediate this multiphysics approach.

\section{Methodology}

Carbon Dioxide Simulation. $\mathrm{CO}_{2}$ sequestration is our first example for coupling reservoir simulation and MD. Following Ennis-King et al. (2005), Slim and Ramakrishnan (2010), and Allen and Sun (2012), a single-phase $\mathrm{CO}_{2}$-transport model is used to describe the density-driven-flow process during $\mathrm{CO}_{2}$ sequestration. In the process, the diffusive and convective transport behavior of the dissolved $\mathrm{CO}_{2}$ in the porous media is described by the transport equation

$$
\frac{\partial(\phi C)}{\partial t}+\nabla \cdot(\mathbf{u} C-\phi D \nabla C)=q, \ldots \ldots \ldots \ldots
$$

where $\phi$ is the porosity, $C$ is the concentration of the dissolved $\mathrm{CO}_{2}, t$ is the time, $\mathbf{u}$ is the Darcy velocity, and $q$ is the source term. Here, we assume $q=0$.

The Boussinesq approximation is used to describe the effects of density change caused by the dissolution of $\mathrm{CO}_{2}$, and the flow is assumed to be incompressible:

$$
\nabla \cdot \mathbf{u}=0 .
$$

With Darcy's law, the velocity can be evaluated with

$$
\mathbf{u}=-\frac{k}{\mu}(\nabla P-\rho \mathbf{g})
$$

where $P$ is the pressure, $k$ is the permeability of the porous media, $\mu$ is the dynamic viscosity of the fluid, $\mathbf{g}$ is the gravitational acceleration vector, and $\rho$ is the density of the fluid. The density $\rho$ is a function of the pressure, temperature, and concentration of $\mathrm{CO}_{2}$.

Different models have been used to describe the impact of $\mathrm{CO}_{2}$ dissolution on the density of the solution. The following linear relation is frequently used (Slim and Ramakrishnan 2010):

$$
\rho(C)=\rho_{0}+\Delta \rho_{\text {sat }} \frac{C}{C_{\text {sat }}},
$$

where $\rho_{0}$ is the density of the pure fluid, $C_{\text {sat }}$ is the saturated concentration of $\mathrm{CO}_{2}$ under certain conditions, and the saturated-density change $\Delta \rho_{\text {sat }}=\rho_{\text {sat }}-\rho_{0}$ is the difference between the density of fluid saturated with $\mathrm{CO}_{2}$ and the pure fluid.

The cell-centered finite-difference method is used to discretize the transport equation (Eq. 1) and flow equations (Eqs. 2 and 3). During each timestep, the transport equation and flow equations are solved alternatively. A backward Euler method is used to solve the transport equation implicitly for better stability and the Portable, Extensible Toolkit for Scientific Computation (known as PETSc) (Balay et al. 2012) is used to solve the resulting nonlinear and linear systems.

MD. In this study, MD simulations are carried out to evaluate the properties of the mixture of $\mathrm{CO}_{2}$ and fluid (water in this study, for simplicity). In the MD simulation, Newton's equations of motion are solved to model a system with $N$ interacting atoms:

$$
m_{i} \frac{\partial^{2} \mathbf{r}_{i}}{\partial t^{2}}=\mathbf{F}_{i}, \quad i=1 \ldots N
$$

where $m_{i}$ is the mass of the $i$ th atom, $\mathbf{r}_{i}$ is the spatial coordinate, and $\mathbf{F}_{i}$ is the force enforced on the $i$ th atom. $\mathbf{F}_{i}$ can be computed as the negative derivative of a potential function $V\left(\mathbf{r}_{1}, \mathbf{r}_{2}, \ldots, \mathbf{r}_{N}\right)$ (van der Spoel et al. 2010):

$$
\mathbf{F}_{i}=-\frac{\partial V}{\partial \mathbf{r}_{i}}
$$

During each timestep, Eqs. 5 and 6 are advanced simultaneously with specified conditions, such as the composition and numbers of molecules, pressure, and temperature, and the movement of all the atoms over time is generated as a trajectory of the system. In the output trajectory files, the positions, velocities, forces, energy, temperatures, and dimensions of the simulation volume can be included (van der Spoel et al. 2010). After running for some time, the system can reach an equilibrium state. The macroscopic properties of the system with the specified conditions can be obtained by averaging the equilibrium trajectory over time. For example, to determine the density of the system under certain temperature and pressure we need for this work, the composition and number of molecules are fixed during the simulation, and then the density of the system can be obtained by tracking the change of the simulation volume over time. The density of the system can be obtained by statistically averaging the density evolution over time. The longer the simulation runs, the more accurate results will be obtained.

In this study, the Lennard-Jones interactions and Coulomb interactions are considered and the following form is used to represent the interaction between two atoms (van der Spoel et al. 2010):

$$
V\left(r_{i j}\right)=V_{L, J}\left(r_{i j}\right)+V_{c}\left(r_{i j}\right)=4 \varepsilon_{i j}\left[\left(\frac{\sigma_{i j}}{r_{i j}}\right)^{12}-\left(\frac{\sigma_{i j}}{r_{i j}}\right)^{6}\right]+f \frac{q_{i} q_{j}}{\varepsilon_{r} r_{i j}},
$$

where $r_{i j}$ is the distance between the two atoms $i$ and $j ; q_{i}$ is the partial charge; $\varepsilon_{i j}$ and $\sigma_{i j}$ are the energy and size parameters for the Lennard-Jones potential, which can be obtained by certain combining rules; $f=1 / 4 \pi \varepsilon_{0}=138.935485$ (van der Spoel et al. 2010); and $\varepsilon_{r}$ is the relative dielectric constant and can be specified by the users.

Following the Optimized Potentials for Liquid Simulations force field (Jorgensen and Tirado-Rives 1988), the following geometric mean mixing rule is used for both parameters:

$$
\begin{aligned}
& \sigma_{i j}=\left(\sigma_{i i} \sigma_{j j}\right)^{1 / 2}, \\
& \varepsilon_{i j}=\left(\varepsilon_{i i} \varepsilon_{j j}\right)^{1 / 2} .
\end{aligned}
$$

In the MD simulation, the interaction potentials of molecules play the key role for the accuracy of the simulation. For example, in the $\mathrm{CO}_{2}$ /water system studied in the paper, three kinds of interactions are involved: water/water, $\mathrm{CO}_{2} / \mathrm{CO}_{2}$, and water/ $\mathrm{CO}_{2}$ interactions. There are many available water and $\mathrm{CO}_{2}$ potential models published. Following Zhang and Duan (2005a), the extended simple point charge (SPC/E) potential is used for water/ water interaction. An optimized $\mathrm{CO}_{2}$ potential model (Zhang and Duan, 2005b) is chosen for $\mathrm{CO}_{2} / \mathrm{CO}_{2}$ interaction. The parameters for the used intermolecular potentials are shown in Table $\mathbf{1 .}$

In this work, we study the properties of fluid mixture under a certain pressure and temperature with a certain molecular composition, so NPT (defined as constant number and composition of molecules, constant pressure, and constant temperature) MD simulations are performed. There are numerous schemes to control the pressure and temperature during the MD simulation. Here, the V-rescale thermostat (Bussi et al. 2007) and Parrinello-Rahman approach (Parrinello and Rahman 1981; Nosé and Klein 1983) are used for the control temperature and pressure, respectively.

Coupling Between the Reservoir Simulation and MD Simulation. The subsurface-fluid properties are dependent on the reservoir conditions, such as pressure, temperature, and fluid composition, whereas the reservoir conditions are also affected by the fluid properties. As a result, the prediction of the fluid properties 


\begin{tabular}{ccc}
\multicolumn{1}{l}{ Water } & & \\
\hline Bond distance $(\mathrm{nm})$ & $\mathrm{d}(\mathrm{O}-\mathrm{H})$ & 0.1 \\
Bond distance $(\mathrm{nm})$ & $\mathrm{d}(\mathrm{H}-\mathrm{H})$ & 0.1633 \\
\hline Atom & $\mathrm{O}$ & $\mathrm{H}$ \\
Molar mass $(\mathrm{g} / \mathrm{mol})$ & 15.9994 & 1.008 \\
$\varepsilon(\mathrm{KJ} / \mathrm{mol})$ & 0.650194 & 0.00000 \\
$\sigma(\mathrm{nm})$ & 0.316557 & 0.00000 \\
$q(\mathrm{e})$ & -0.8476 & 0.4238 \\
$\mathrm{CO}_{2} \quad$ & $\mathrm{~d}(\mathrm{C}-\mathrm{O})$ & 0.1163 \\
\hline & $\theta(\mathrm{O}-\mathrm{C}-\mathrm{O})$ & 180 \\
\hline Bond distance $(\mathrm{nm})$ & $\mathrm{C}$ & 0 \\
Bond angle (degrees) & 12.0110 & 15.9994 \\
Atom & 0.2398 & 0.6872 \\
Molar mass $(\mathrm{g} / \mathrm{mol})$ & 0.27918 & 0.30000 \\
$\varepsilon(\mathrm{KJ} / \mathrm{mol})$ & 0.5888 & -0.2944 \\
\hline$\sigma(\mathrm{nm})$ & &
\end{tabular}

Table 1-The potential models used for $\mathrm{CO}_{2}$ and $\mathrm{H}_{2} \mathrm{O}$ in this study.

under reservoir conditions plays a key role in the reservoir simulation. In the work, MD simulations are used to evaluate the fluid properties under certain reservoir conditions. Here, a coupler is introduced to make the reservoir simulation with RST and the MD simulation (MDS in Fig. 1) with Gromacs (van der Spoel et al. 2010) work simultaneously.

The flow chart of the presented computational framework is shown in Fig. 1. As shown in the flow chart, during each timestep, fluid-property values under certain conditions, such as the density of the $\mathrm{CO}_{2}$ /water mixture under different pressure and $\mathrm{CO}_{2}$ concentration in the work, will be requested by the RST, and the requests will be sent to the coupler. The coupler will check the lookup table first to find if the requests can be satisfied with the existing data in the lookup table. If yes, the coupler will just return the requested fluid-property values to RST. If some of the requests cannot be satisfied, then MD simulations will need to be performed. The coupler will determine how many MD simulations need to be performed and the parameters of the MD simulations. The scheduler will perform the MD simulations in an efficient way to optimize the performance and maximize the computing-resource usage. The results obtained from the MD simulations are used to update the lookup table. The maintenance of the lookup table and the scheduler play the key roles in the operation of the coupler.

The lookup table is established with an irregular $n$-dimensional mesh. The keys of the lookup table are represented with the node coordinates of the underlying mesh, which are $n$-tuples of reservoir conditions, such as pressure and temperature. The values of the lookup table are the physical-property values. As a result, the

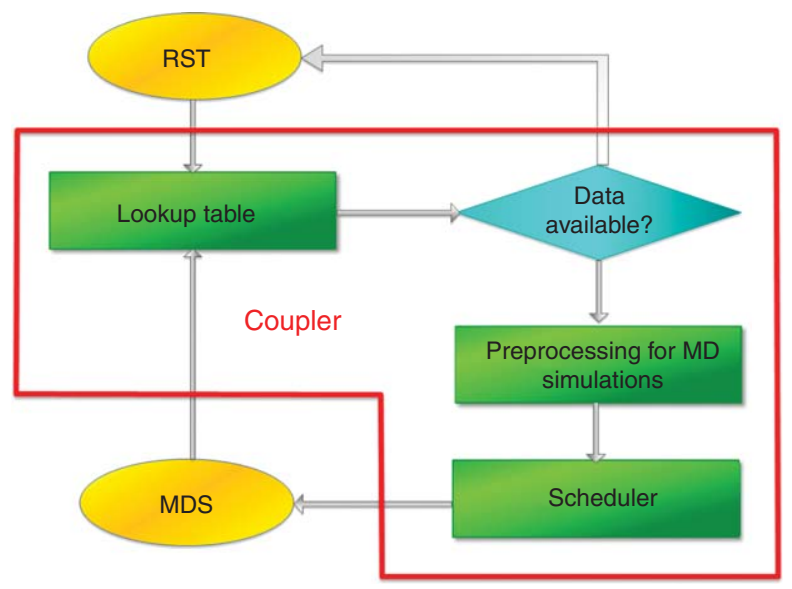

Fig. 1-The flow chart of the computational framework.
$n$-D lookup table represents the discrete evaluation of a function with $n$ variables:

$$
y=F\left(x_{1}, x_{2}, \ldots, x_{n}\right), \ldots \ldots \ldots \ldots \ldots
$$

where $x_{1}, x_{2}, \ldots, x_{n}$ are the input physical parameters and $y$ is the returned physical-property value, which is the density of the $\mathrm{CO}_{2} /$ water mixture in the work. For the given input physical conditions, the lookup table will return the required value by looking up or interpolating with the surrounding node values. At the moment, the nearest-neighbor and linear interpolation are supported by the lookup table. The following functionalities are provided for the maintenance of the lookup table:

- The lookup table can be initialized with a disk file and dumped to a file. The function is not only required by the checkpoint support, but also provides the fundamental for loose coupling between RST and MD simulation with disk files.

- Given a group of input physical parameters, the smallest box surrounding the point can be found very efficiently. If the bounding box is found successfully, depending on the size of the box, the lookup table can either return an interpolated value with the values on the surrounding nodes or return a flag to indicate that MD simulation is required. If the given parameters are out of the range that the lookup table covers, a flag for a MD-simulation request will also be returned.

- Given a point specified by physical parameters and a property value, the lookup table can be updated by inserting the point into the lookup table or replacing the existing point value in the table.

Here, because of the complexity of the reservoir simulations, the dimension of the lookup table can be higher than four. Therefore, we cannot simply use a general mesh-management library that supports up to three dimensions, such as MOAB: MeshOriented datABASE (Yan et al. 2012). Here, the lookup table is implemented with a nested $\mathrm{C}++$ map, which is usually provided with a search tree in most compilers, and it is very efficient to insert, delete, and locate an element.

The reservoir simulation is parallelized on the basis of domain decomposition, with each process handling a subdomain. During each timestep, each process may get a list of requests that cannot be satisfied by the lookup table. It is not desirable to perform MD simulation to fulfill each request. Therefore, all the requests will be sent to the master process first. The master process will gather all the information and determine the number and input parameters of the required MD simulations so that the lookup table can satisfy the requests by updating with the results from the MD simulations. 


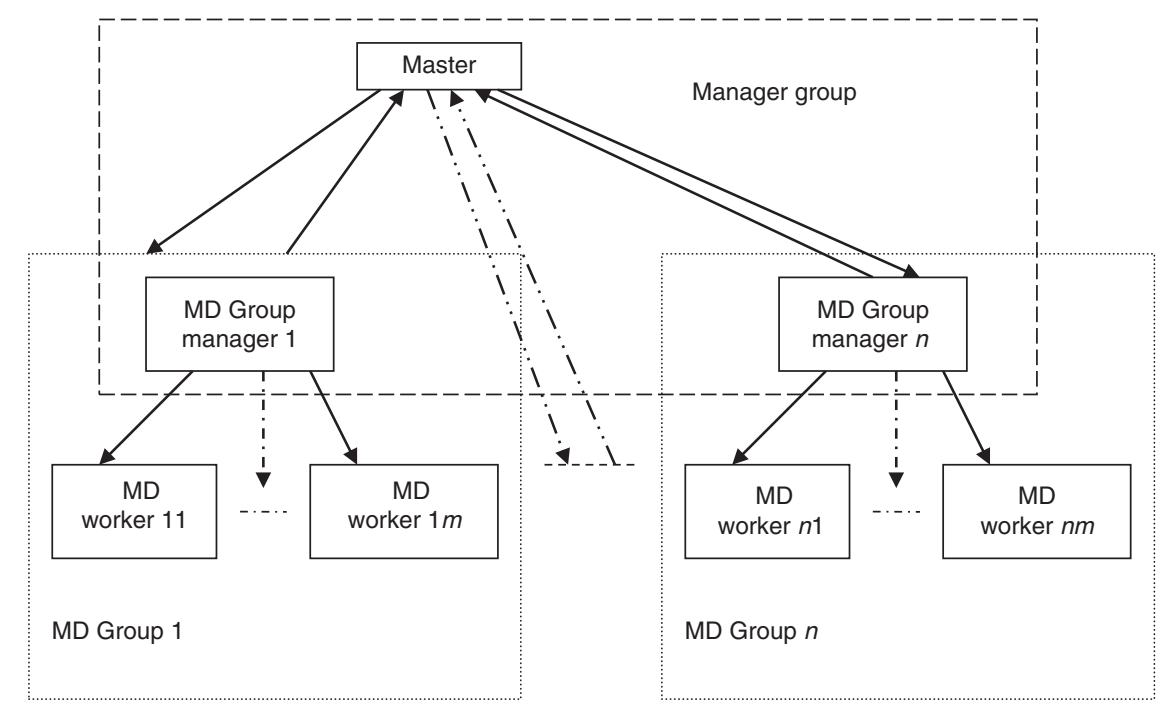

Fig. 2-Management of the scheduler.

As a result of the scale and properties of MD simulation, a typical MD simulation is much more time-consuming than reservoir simulation and can usefully engage fewer parallel resources at the same time. Therefore, it is undesirable to perform one MD simulation with all the message passing interface (MPI) processes on the large-scale HPC platforms one after another. Here, the MPI processes are split into multiple groups. One MD simulation will be performed on one group. There may be more than one MD simulation that will run on one group, and each MD simulation requires multiple steps. Some of the steps are parallel, whereas the other steps, mostly the preprocessing and post-processing steps, are serial. Therefore, for each group, a manager is introduced to manage the performance of the MD simulations. On the basis of these considerations, the scheduler assigns each process one of three roles: master, MD group manager, and MD worker, and a two-level management is used, as shown in Fig. 2.

There is one and only one master process. The master process maintains a latest version of the lookup table, keeps a list of active MD requests that are being handled, and tracks the status of each MD group. After all MD requests are handled, the master process broadcasts the latest lookup table to all processes. The main responsibilities of the master process include the following.

1. Collecting MD requests from all processes.

2. For each MD request, deciding if the request is granted or ignored. The basic principle is that for the MD requests

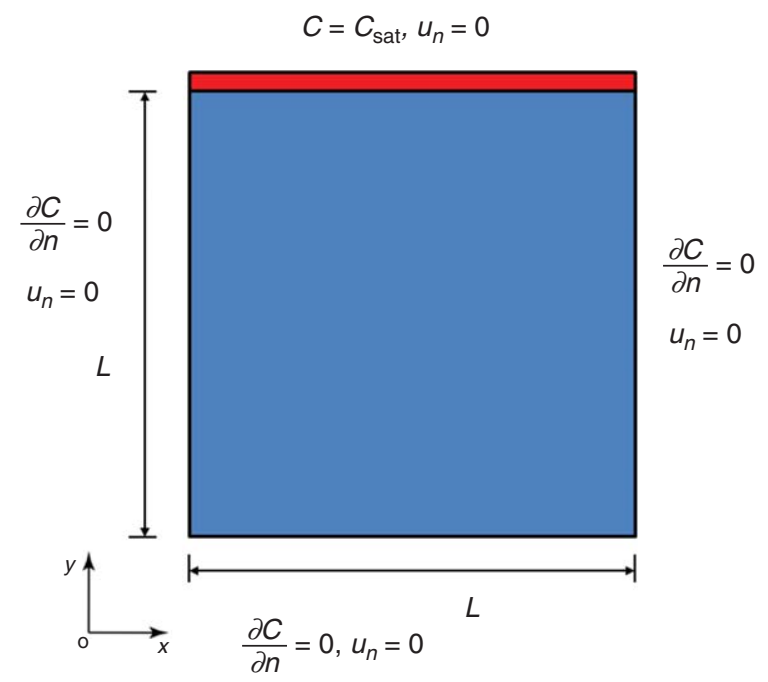

Fig. 3-The configuration for the $\mathrm{CO}_{2}$ simulation (Allen and Sun 2012). where their distance in subsurface-parameter space is smaller than a threshold value, only one MD request will be granted and the other requests will be all ignored.

3. For each granted MD request:

a. Generate an entry with the physical parameters of the request and a dummy property value in the lookup table.

b. Dispatch the granted MD request to an available MD group. In the case that there is no available MD group, wait until a MD group becomes available.

c. Collect the returned value from the MD simulation and update the lookup table by replacing the dummy value with the returned value.

4. After all the MD requests have been handled, broadcasting the latest lookup table to all the processes.

All the MD simulations are performed by MD group managers and the MD workers in their groups. A MD group manager not only receives MD requests and returns the results of the simulations to the master process, but also performs the serial tasks to handle the necessary preprocessing and post-processing works, including initialization of the coordinates of the atoms, generation of binary-configuration files for MD running, and evaluation of fluid properties with the output trajectory files. The kernel parallel-computational task of MD simulations is performed by MD group managers and their workers together.

The job of MD workers is to perform MD simulations under the management of MD group managers. An MD worker starts the MD simulation when requested by the manager.

The determination of the size of an MD group or the number of MD groups is dependent on the scalability analysis. The smaller the MD group is, the less the communication will cost during the MD simulation. However, the smaller the MD group is, the greater the number of MD groups will be. When there are not enough MD requests for all MD groups, some of the MD groups will be idle. To achieve optimal performance and resource usage, the scheduler is designed to be able to adjust the size of the MD group dynamically according to the number of MD requests.

With this design of lookup table and scheduler, the coupler couples RST and MD simulation efficiently.

\section{Results and Discussion}

Carbon Dioxide Simulation. The configuration of the simulation is shown in Fig. 3 (for clarity, a 2D illustration is used). In the figure, $n$ indicates the outward normal direction of the boundary surface, and $u_{n}$ and $\frac{\partial C}{\partial n}$ represent the Darcy velocity and concentration gradient in the normal direction on the boundary, respectively. Initially, the domain is saturated with water and the flow in 


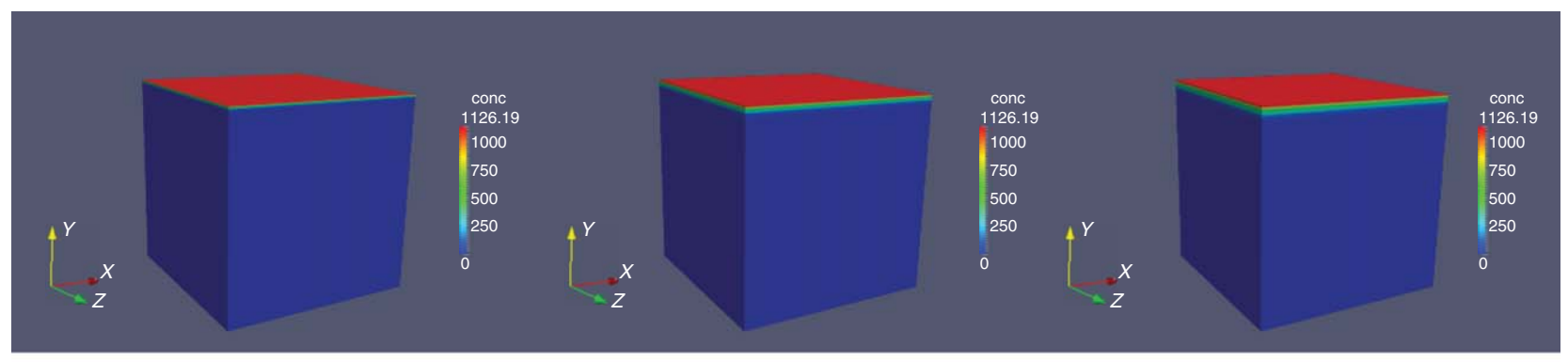

(a) 0.25 days

(b) 1.25 days

(c) 2.25 days

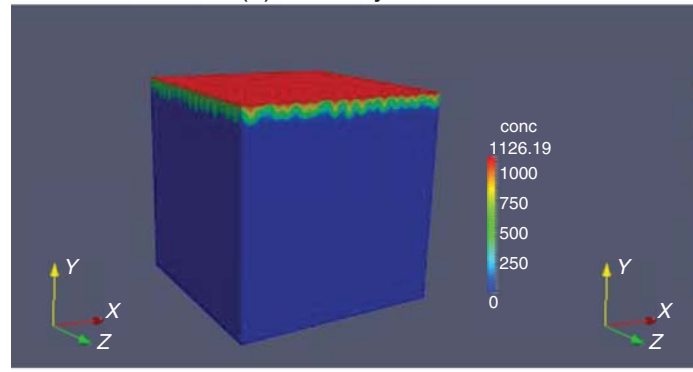

(d) 4.75 days

(e) 6.5 days
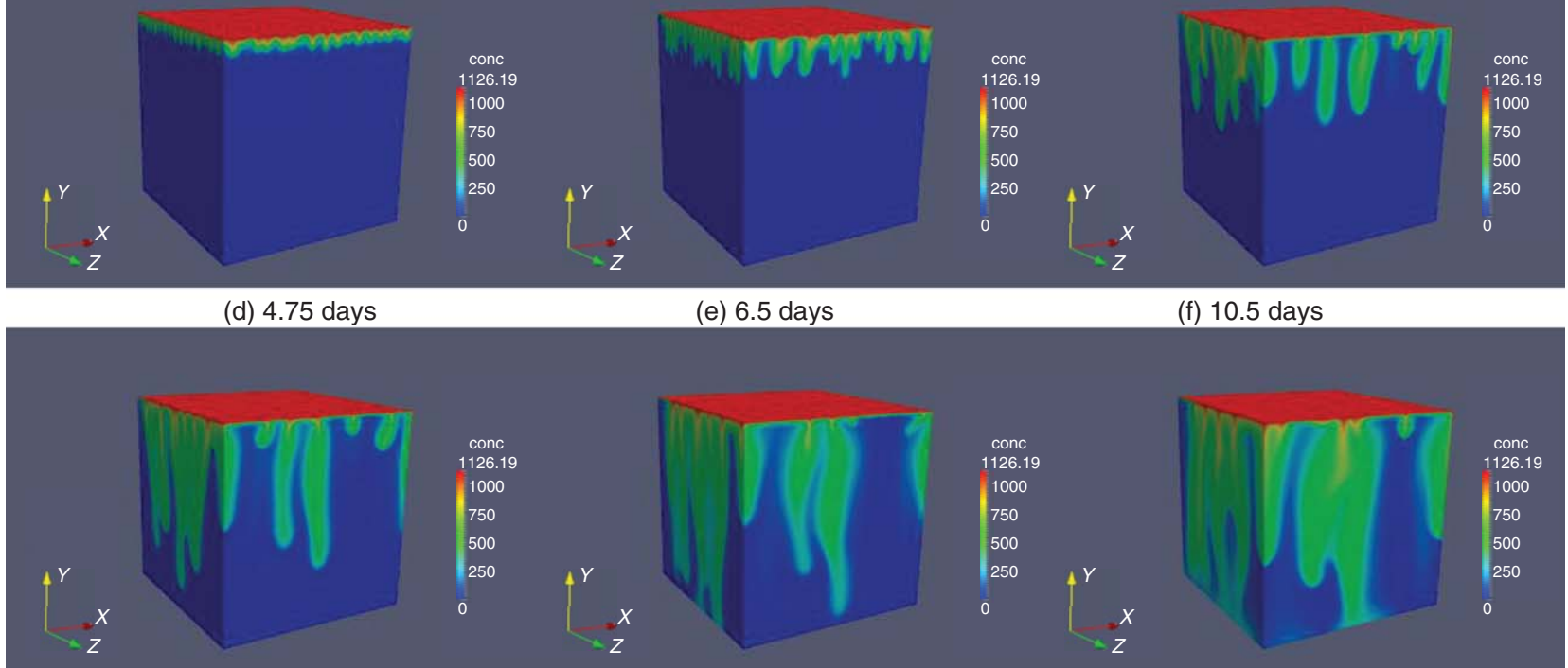

(g) 14.5 days
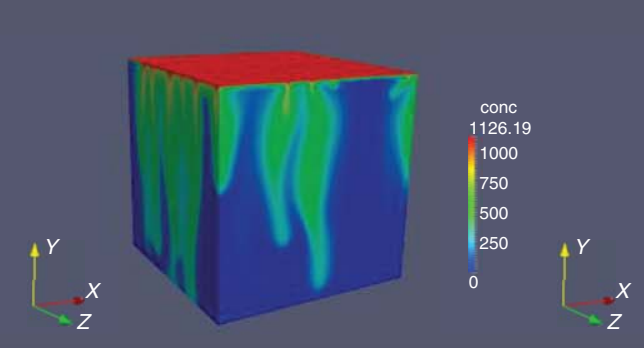

(f) 10.5 days

Fig. 4-The $\mathrm{CO}_{2}$-concentration profile over time with the base-saturated-density change.

the domain is stationary. No-flow boundary conditions are enforced for all the boundaries. The top boundary is saturated with dissolved $\mathrm{CO}_{2}$. The configuration is widely used for validation and comparison of $\mathrm{CO}_{2}$-sequestration modeling (Pruess and Zhang 2008; Pau et al. 2010; Allen and Sun 2012).

With this configuration and concentration on the top boundary constant over time, the quantity of $\mathrm{CO}_{2}$ that diffuses into the underlying fluid can be computed analytically with a complementary error function (Pruess and Zhang 2008; Clark 2009; Allen and Sun 2012). With this function, the concentration with distance $y$ from the boundary at time $t$ can be evaluated as

$$
C_{\mathrm{CO}_{2}}(y, t)=C_{\mathrm{CO}_{2}, y=0} \operatorname{erfc}\left(\frac{y}{2 \sqrt{D t}}\right) \text {, }
$$

where $C_{\mathrm{CO}_{2}}(y, t)$ is the concentration at depth $y$ and time $t$. $C_{\mathrm{CO}_{2}, y=0}$ is the concentration at the top boundary, and $D$ is the

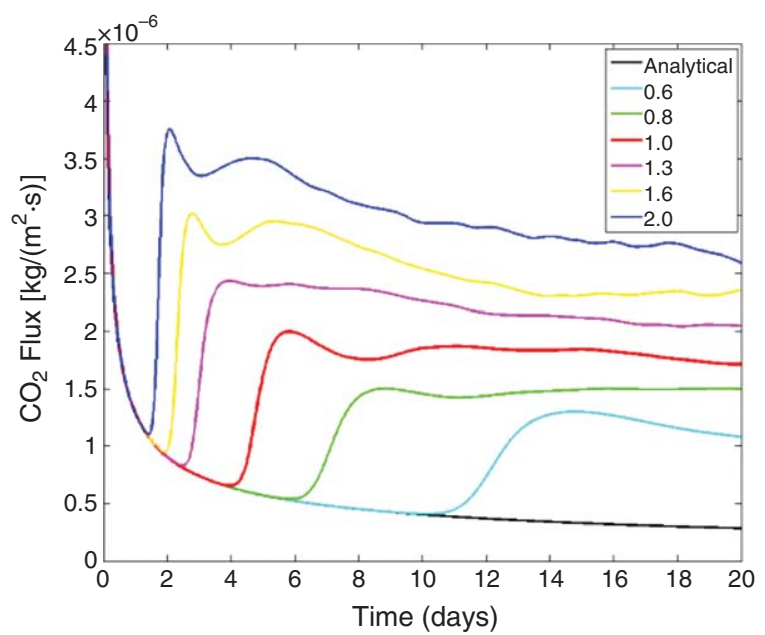

(a)

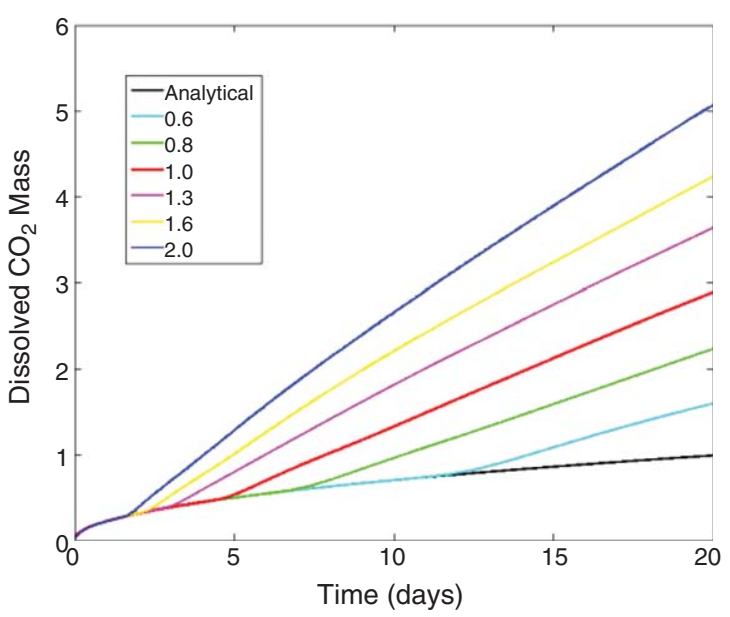

(b)

Fig. 5-The $\mathrm{CO}_{2}$ mass flux through the top boundary (a) and the accumulated mass of dissolved $\mathrm{CO}_{2}$ (b) over time with different saturated-density changes. 


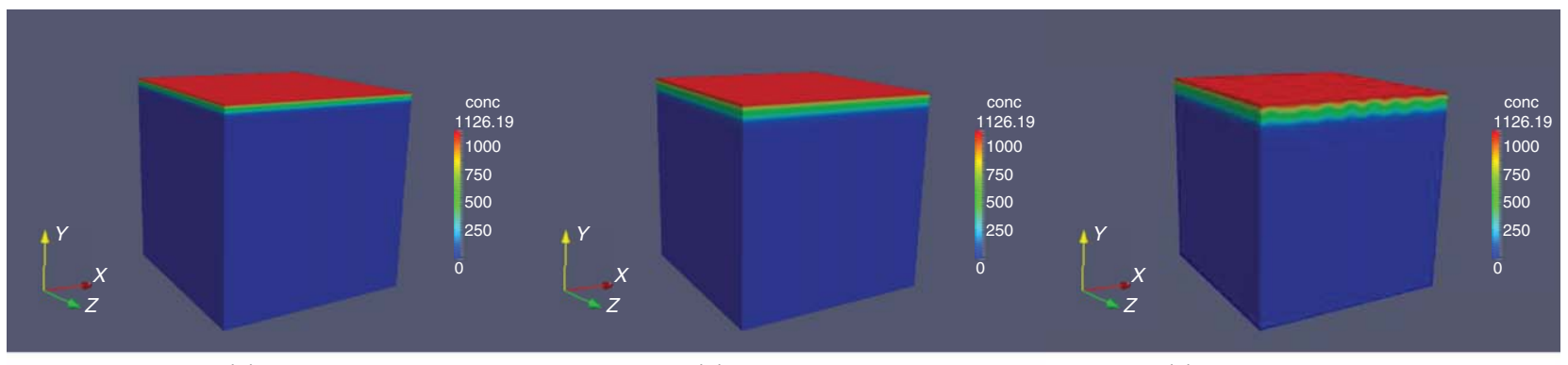

(a) 4.25 days

(b) 6.5 days

(c) 10.5 days

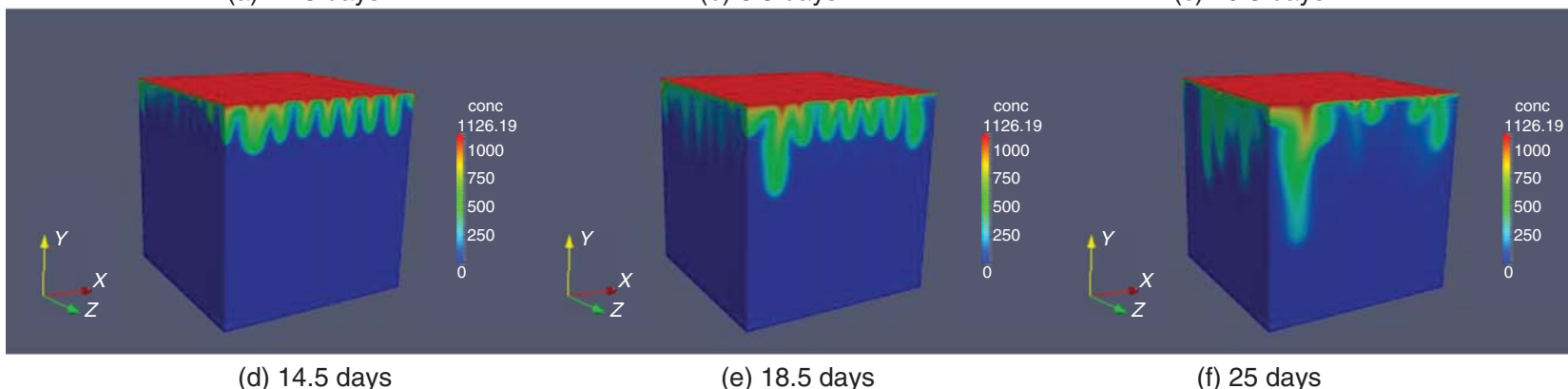

Fig. 6 -The $\mathrm{CO}_{2}$-concentration profile over time with $0.6 \Delta \rho_{\text {sat }}$.

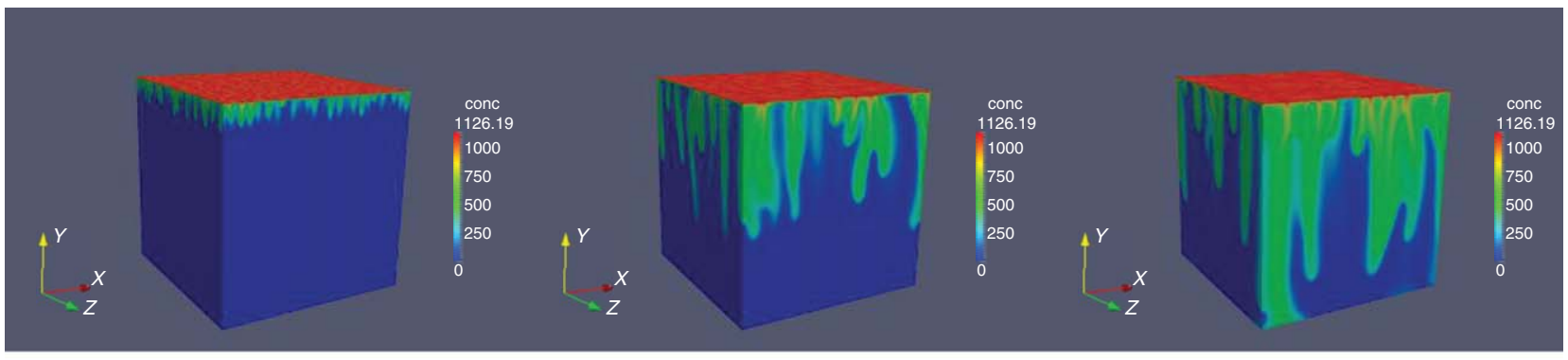

(a) 4.25 days

(b) 6.5 days

(c) 10.5 days
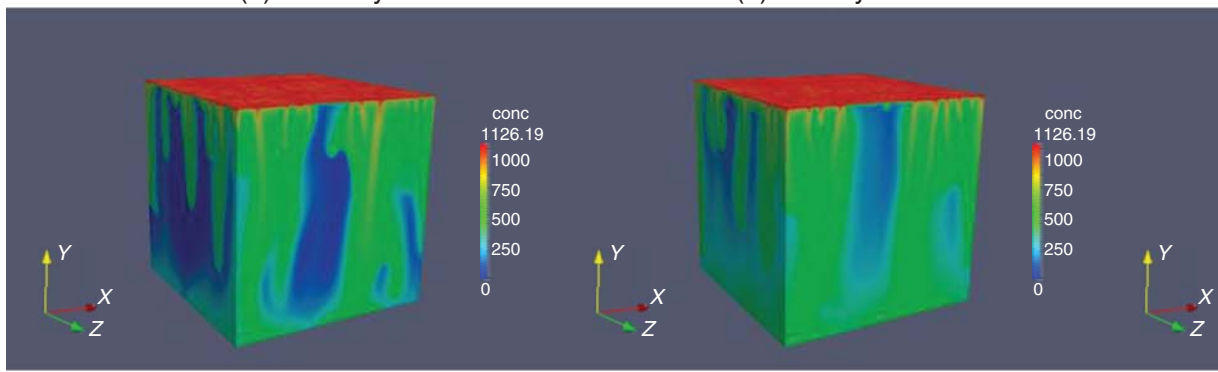

(d) 14.5 days

(e) 18.5 days

(f) 25 days

Fig. 7-The $\mathrm{CO}_{2}$-concentration profile over time with $2.0 \Delta \rho_{\text {sat- }}$

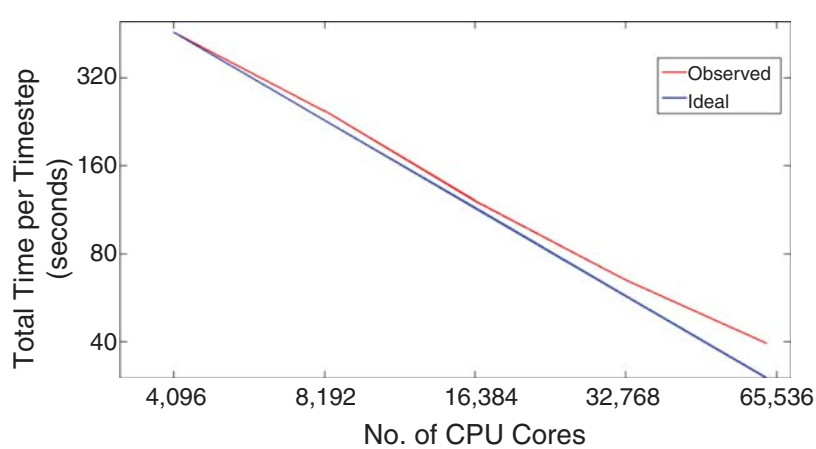

Fig. 8-Strong scaling result of RST on Shaheen. diffusion coefficient between $\mathrm{CO}_{2}$ and water. $\operatorname{erfc}(x)=1-\operatorname{erf}(x)$ is the complementary error function, and

$$
\operatorname{erf}(x)=\frac{2}{\sqrt{\pi}} \int_{0}^{x} \exp \left(-u^{2}\right) \mathrm{d} u .
$$

With Eqs. 11 and Eq. 12, the flux of the $\mathrm{CO}_{2}$ through the top boundary is computed as

$$
f_{\mathrm{CO}_{2}}=-\left.D \frac{\partial C_{\mathrm{CO}_{2}}}{\partial y}\right|_{y=0}=C_{\mathrm{CO}_{2}, y=0} \sqrt{\frac{D}{\pi t}}
$$

and the total amount of $\mathrm{CO}_{2}$ dissolved into the underlying fluid can be evaluated with 


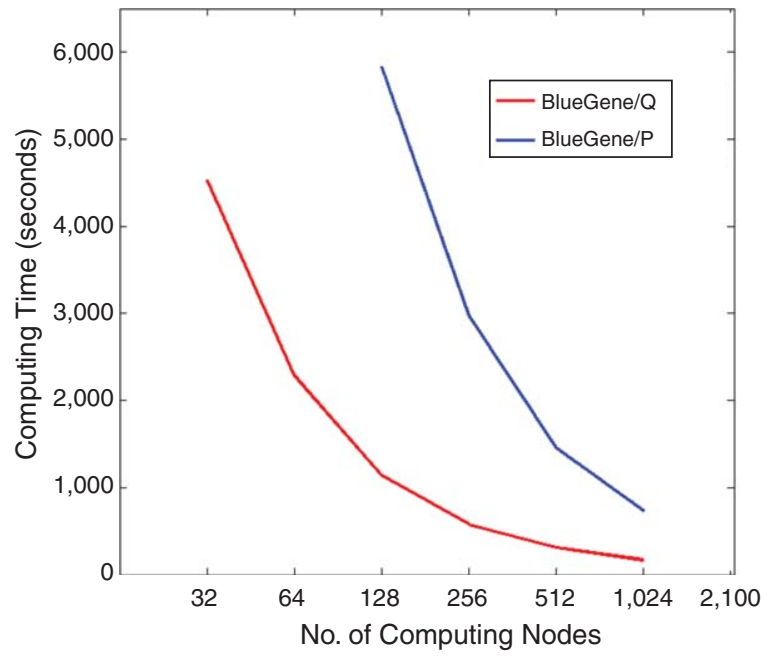

(a)

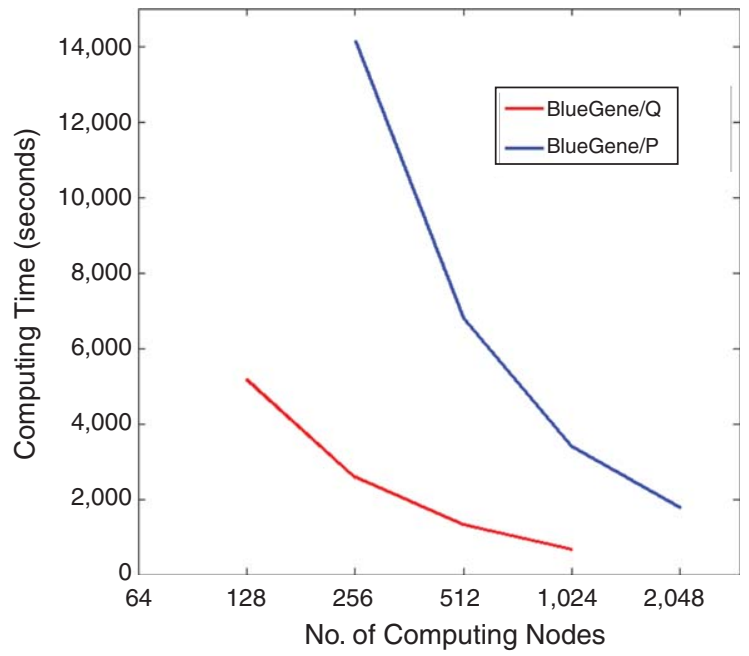

(b)

Fig. 9-The results of scaling test of RST on BlueGene/Q vs. BlueGene/P per nodes (a) mesh size $512^{3}$ and (b) mesh size $768^{3}$.

\begin{tabular}{ccccc} 
This work & $\begin{array}{c}\text { Zhang and Duan } \\
(2005 \mathrm{a})\end{array}$ & $\begin{array}{c}\text { Berendsen } \\
\text { et al. (1987) }\end{array}$ & $\begin{array}{c}\text { Paschek } \\
(2004)\end{array}$ \\
\hline$\rho\left(\mathrm{g} / \mathrm{cm}^{3}\right)$ & $0.99863 \pm 0.00007$ & $0.997 \pm 0.008$ & 0.998 & 0.9986 \\
\hline
\end{tabular}

Table 2-MD results with SPC/E water molecule model.

$$
M_{\mathrm{CO}_{2}}=2 C_{\mathrm{CO}_{2}, y=0} \sqrt{\frac{D t}{\pi}} . \ldots \ldots \ldots \ldots \ldots
$$

With Eqs. 13 and 14, the pure diffusive behavior is described analytically. As a result, the computation with the equations can be used to validate the simulation results before the convective mixing happens.

Following Pruess and Zhang (2008), Pau et al. (2010), and Allen and Sun (2012), the following parameters (under pressure of 100 bar and temperature of $318 \mathrm{~K}$ ) and the linear relation (Eq. 4) for density evaluation are used in the simulation, with $\mu=5.947 \times$ $10^{-4} \mathrm{~Pa} / \mathrm{s}, \rho_{0}=994.56 \mathrm{~kg} / \mathrm{m}^{3}, C_{\text {sat }}=1126.19 \mathrm{~mol} / \mathrm{m}^{3}, \Delta \rho_{\text {sat }}=$ $10.45 \mathrm{~kg} / \mathrm{m}^{3}, g=9.81 \mathrm{~m} / \mathrm{s}^{2}, D=2 \times 10^{-9} \mathrm{~m}^{2} / \mathrm{s}, \phi=0.3$, and $k=1 \times 10^{-11} \mathrm{~m}^{2}$.

Our 3D simulations are made possible by parallel computation. A nonuniform Cartesian product mesh is used, with a veryfine resolution near the top boundary to accurately simulate the diffusion behavior there (Pruess and Zhang 2008). The final resolution of the used mesh is $160 \times 320 \times 160$.

The result with this configuration is shown in Fig. 4. As seen at the early stage, only uniform diffusion happens through the top boundary, and then some instability can be observed near the diffusion layer (Fig. 4d). As time progresses, the instability becomes more and more significant. Finally a gravitational fingering phe-

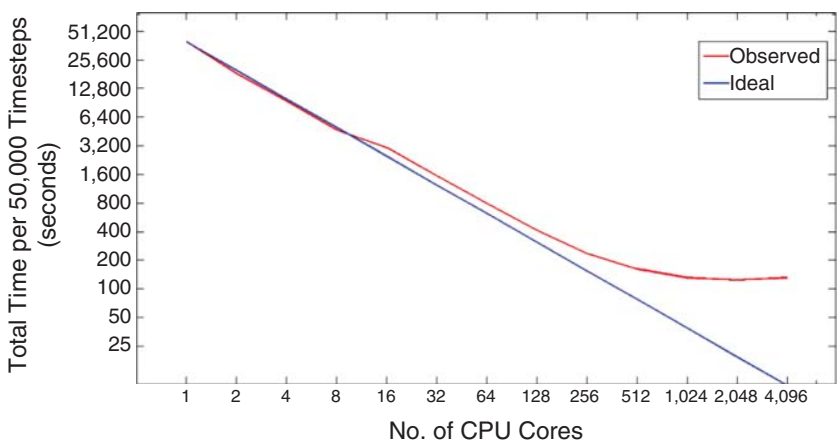

Fig. 10-Strong scaling result of Gromacs (van der Spoel et al. 2010) on BlueGene/P. nomenon is observed, which indicates that convective mixing happens. This is because of the greater density of the $\mathrm{CO}_{2} /$ water solution. With the convective mixing, the dissolution of the $\mathrm{CO}_{2}$ into the underlying fluid is greatly enhanced, which can be seen in Fig. 5 (red line). In Fig. 5, the lines with different colors indicate the $\mathrm{CO}_{2}$ mass flux through the top boundary (Fig. 5a) and the accumulated mass of dissolved $\mathrm{CO}_{2}$ in the whole domain (Fig. $5 b)$ with different saturated-density changes. The deviation from the analytical results indicates the onset of convective mixing (Pruess and Zhang 2008; Pau et al. 2010). Before the onset of the fingering instability, the simulated results agree well with the 1D analytical results, with which the used $\mathrm{CO}_{2}$ model and implementation are well-verified.

Simulations with different saturated-density changes are also performed to investigate the effect of saturated-density change on the $\mathrm{CO}_{2}$-dissolution process (Allen and Sun 2012). On the basis of the $\Delta \rho_{\text {sat }}$ used in the previous simulation, we set the saturated-density change to be $0.6,0.8,1.3,1.6$, and $2.0 \Delta \rho_{\text {sat }}$, respectively, and the results are shown in Fig. 5. As shown in the results, with smaller $\Delta \rho_{\text {sat }}$, the onset of the convective mixing is delayed, and with larger density changes, the dissolution of $\mathrm{CO}_{2}$ into the fluid is enhanced. The results show that the density change plays a key role in the occurrence of the convective mixing and the dissolution process.

The resulting $\mathrm{CO}_{2}$-concentration profile over time with two different saturated-density changes, 0.6 and $2.0 \Delta \rho_{\text {sat }}$, are shown in Figs. 6 and 7, respectively.

Computational Scalability of the Reservoir Model. A scalability test is performed by use of the previous example for the $\mathrm{CO}_{2}$ simulation module. The resolution of the mesh for the scalability test is $1,000 \times 1,000 \times 1,000$, with which one billion cells are involved in the solution. The biconjugate gradient-stabilized method in the Portable, Extensible Toolkit for Scientific Computation (Balay et al. 2012) is used as the linear solver along with a block-Jacobi preconditioner that applies an incomplete LUdecomposition with zero fill-in [ILU(0)] for each block. We ran the simulation for 10 timesteps on King Abdullah University of Science and Technology's Shaheen supercomputer (BlueGene/P) and the result is shown in Fig. 8, from which we can see the scaling behavior is good with up to 65,536 central-processing-unit 
The number of the MD simulations

The number of the computing nodes used

The mesh size of the reservoir simulation

Computing resource used by the reservoir simulation

Wall-clock computing time for the reservoir simulation

Computing resource used by each MD simulation

Each MD simulation takes approximately

Number of MD simulations can be performed simultaneously

Total wall-clock computing time for the MD simulations
$11 \times 9=99$

1,024 nodes (4096 CPU cores)

$160 \times 320 \times 160$

1,024 nodes (4096 MPI tasks)

$00: 48$

64 nodes ( $256 \mathrm{MPI}$ tasks)

$2: 49$

16

$19: 41$

Table 3-The computing resource and computing time used in the simulation.

(CPU) cores (all 16 racks of Shaheen). We also tried the algebraic multigrid preconditioners from the Hypre/BoomerAMG and Trilinos/ML solvers, although they are not as robust and scalable as the block-Jacobi. These solvers show much faster convergence while taking longer total wall-clock time when they do not run out of memory. Sometimes, they request more memory than can be handled on our BlueGene/P nodes. Similar results have been reported in Mills et al. (2009).

Scaling tests are also performed on the latest BlueGene/Q system at the IBM T.J. Watson Research Center, Yorktown Heights, New York, USA. Because only one rack of BlueGene/Q (1,024 nodes) was available during the work, two smaller problems with $512^{3}$ (Fig. 9a) and $768^{3}$ (Fig. 9b) mesh, respectively, are used in the tests, with $32-1,024$ nodes (512-16,384 cores). The configurations of the program on both systems are the same and no additional optimization is performed on the BlueGene/Q system. We ran the simulation for 20 time steps and the results are shown in Fig. 9. From the results, we see that, even without use of the single instruction, multiple data (SIMD) (vectorization) and hardware threads (four hardware threads for each core), reservoirsimulation toolbox achieves performance improvement of approximately five times for BlueGene/Q over BlueGene/P. It can be anticipated that better performance can be obtained on BlueGene/ $\mathrm{Q}$ by enabling hardware threading and SIMD or using other optimization techniques.

Molecular Dynamics. A four-step scheme, which includes energy minimization, NVT (defined as constant number and composition of molecules, constant volume, and constant temperature) equilibrium, NPT equilibrium, and production running, is used for the MD simulations. The number of time steps for NVT equilibrium, NPT equilibrium, and production running are 100,000, 500,000 , and 1.6 million, respectively. To validate our MD simulation, a comparison with some prior works is conducted. The temperature and pressure are set to be $298.15 \mathrm{~K}$ and $1.01325 \mathrm{bar}$, respectively. The comparison result is shown in Table 2 , with which the accuracy of our MD simulation is well-demonstrated.

A scalability test is performed for the MD simulation with Gromacs (van der Spoel et al. 2010); 10,000 molecules (water and

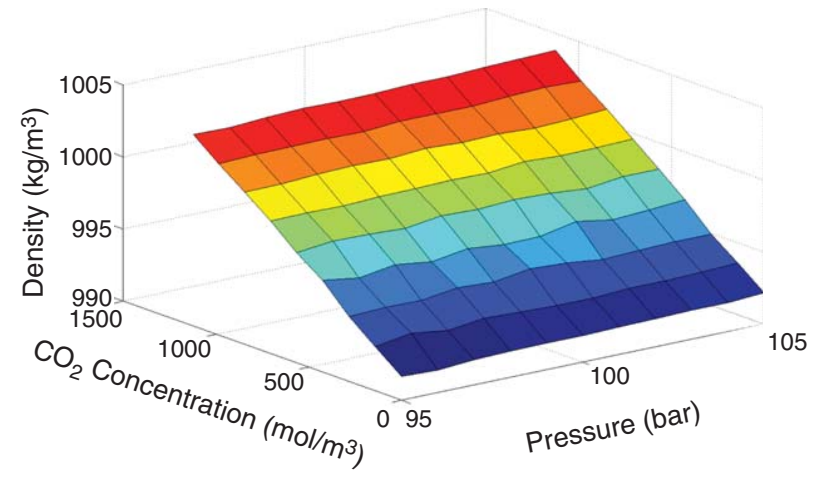

Fig. 11-The density of $\mathrm{CO}_{2}$ /water mixture over different pressures and $\mathrm{CO}_{2}$ concentrations.
$\mathrm{CO}_{2}$ ) or 30,000 atoms are used in the simulation and the timing is modeled after 50,000 MD simulation time steps. The result is shown in Fig. 10. From the result we can see that with the 10,000 molecules, Gromacs can be well-scaled with up to $256 \mathrm{CPU}$ cores (64 nodes on BlueGene/P). There is a deviation of performance with 16 CPU cores, which is because of the employment of multiple-program multiple-data (MPMD) particle-mesh Ewald (PME) parallelization technique (Hess et al. 2008) for the long-range electrostatics calculation. The basic idea of MPMD PME parallelization is to reserve a certain number of processors to handle the PME computation. As a result, the number of MPI tasks involved in PME calculation is reduced and so is the workload because of global communications. Although this causes some degeneration of the performance with $16 \mathrm{CPU}$ cores, Gromacs performs better with more than 128 cores by use of MPMD PME parallelization in our experiments. With more than 256 cores for the simulation, Gromacs can fail without the use of the MPMD PME parallelization. In a separate effort at King Abdullah University of Science and Technology, we are seeking a massive improvement in the scalability of MD codes, and Gromacs, in particular, by replacing the PME computation of the electrostatic force with the fast multiple method.

Result With Coupling MD and Reservoir Simulation. A typical MD simulation is very time-consuming, so it is undesirable to stop the reservoir simulation to perform the MD simulation frequently. To reduce the times for starting MD simulations, we estimate the possible ranges of the parameters required in the simulation. On the basis of the estimation, MD simulations are carried out to generate the lookup table before the reservoir simulation. When the estimation is not good enough and parameters beyond the estimated range are requested, the performance of MD simulations will be still required.

A simulation with both MD simulation and reservoir simulation in a single run is performed. All the parameters are the same with the simulation shown in Fig. 4, except that the density of the fluid mixture is evaluated with the interpolation of the lookup table from the MD simulations. For the whole simulation, one rack on Shaheen (BlueGene/P) with 4,096 CPU cores is used. The simulation begins with an empty lookup table. As shown in Table 3, 99 MD requests are sent to initialize the lookup table. For each MD simulation, on the basis of the scalability analysis in the previous section and the total number of the MD simulations, $256 \mathrm{CPU}$ nodes are used and $16 \mathrm{MD}$ simulations can be performed in the same time. The MD simulations take 19 hours and 41 minutes in total.

The resulting density of the $\mathrm{CO}_{2}$ /water mixture over different concentration of $\mathrm{CO}_{2}$ and pressure with the $99 \mathrm{MD}$ simulations is shown in Fig. 11. The range of the pressure is set to be from 95 to $105 \mathrm{bar}$. The range of the concentration is from 0 to $1126.19 \mathrm{~mol} /$ $\mathrm{m}^{3}$. The temperature is fixed at $318 \mathrm{~K}$. From the result, we can see a nearly linear relation between the density of the mixture and $\mathrm{CO}_{2}$ concentration, which has been discussed in Slim and Ramakrishnan (2010) and Ennis-King et al. (2005). Besides, the concentration of $\mathrm{CO}_{2}$ plays a very important role in the density change of the fluid mixture, and the density change with pressure is much less significant.

With the results from the MD simulations, a 2D lookup table is generated. The reservoir simulation derived from the generated 


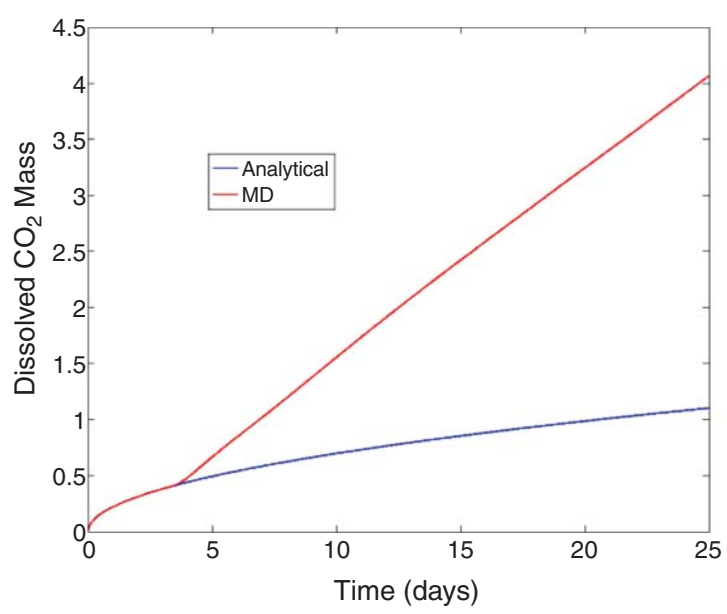

(a)

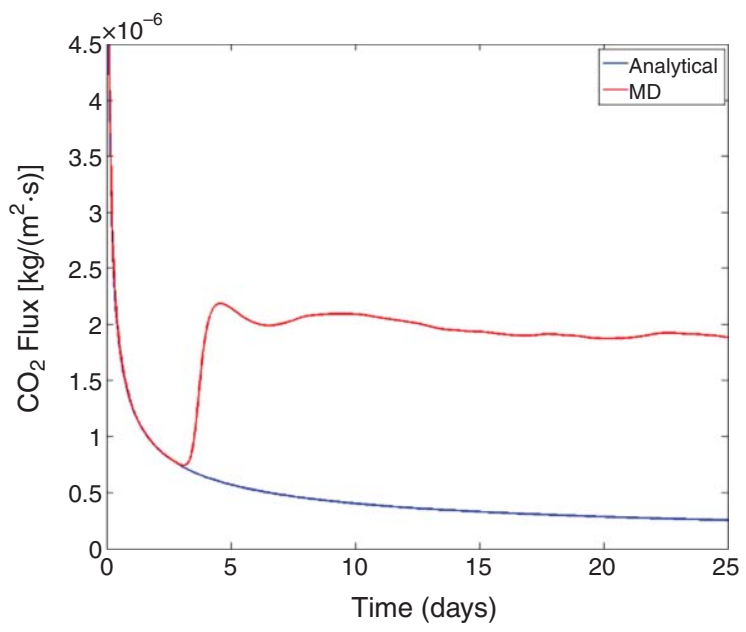

(b)

Fig. 12-The results on the basis of density computation from MD simulations: (a) the accumulated mass of dissolved $\mathrm{CO}_{2}$ and (b) the $\mathrm{CO}_{2}$ mass flux through the top boundary.

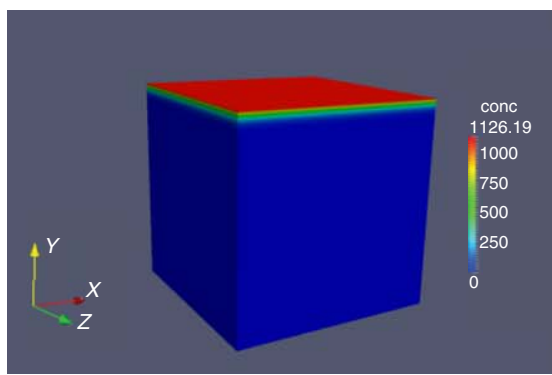

(a) 3 days

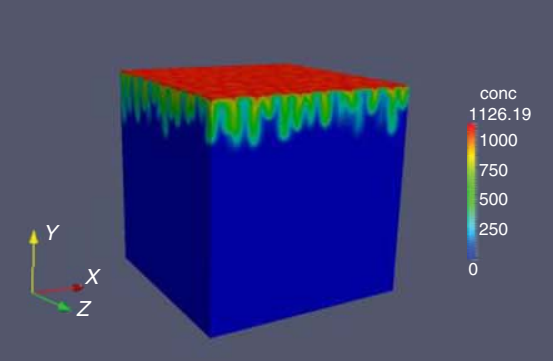

(b) 6 days

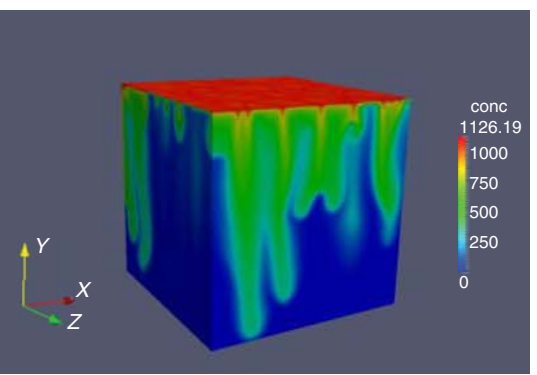

(c) 15 days

Fig. 13-The resulting $\mathrm{CO}_{2}$-concentration profile over time on the basis of density computation from MD simulations.

lookup table is conducted, which takes 48 minutes in total. The resulting accumulated mass of dissolved $\mathrm{CO}_{2}$ in the domain and $\mathrm{CO}_{2}$ mass flux through the top boundary are shown in Fig. 12 (red curves). The analytical result (blue curves in Fig. 12) indicates the accumulation and mass flux of $\mathrm{CO}_{2}$ caused by pure diffusive transport, given by Eqs. 14 and 13, respectively. Through the comparison between the result dependent on the density computation from MD simulations and the analytical result, the pure diffusive process at the early stage is validated and the onset of the convective mixing caused by density change can be observed at the point in time when the simulated curves (red) diverge from the analytical curves (blue). The $\mathrm{CO}_{2}$-concentration profile over time is shown in Fig. 13. As shown in Table 3, the MD simulations take the most of the time. However, because we can dump the lookup table to the disk and use it for the next running with the same configuration, we do not need to perform the MD simulations within the range of the pressure and concentration used this time again.

\section{Conclusions}

A parallel computational framework for $\mathrm{CO}_{2}$-sequestration simulation by coupling reservoir simulation and MD on massively parallel high-performance-computing systems is introduced. Experimental results for different parts of the framework are presented to demonstrate the performance and capacity of the proposed framework.

The proposed example is our first example to couple the reservoir simulation and MD simulation, and only the density of the fluid mixture is evaluated with MD simulation. Our results are preliminary and more experiments and improvements remain to be performed for practical applications. One major direction of the future works is to extend the framework to handle more complicated physics, such as compositional flow, and the evaluation of more properties with different fluid compositions. In our current work, only equilibrium MD simulation is used. To incorporate the prediction of dynamic and transport properties (Firouzi and Wilcox 2012; Firouzi and Wilcox 2013) to handle more-complicated phenomena, nonequilibrium MD will be required.

\section{Acknowledgments}

This publication was derived from the work supported in part by the project titled "Parallel Multiscale Simulation of Multicomponent Multiphase Flow: From Darcy Scale to Molecular Scale," funded by King Abdullah University of Science and Technology's Global Research Partnership Program.

\section{References}

Allen, R. and Sun, S. 2012. Carbon Dioxide Sequestration: Modeling the Diffusive and Convective Transport under a $\mathrm{CO}_{2} \mathrm{Cap}$. Presented at the SPE Saudi Arabia Section Technical Symposium and Exhibition, AlKhobar, Saudi Arabia, 8-11 April. SPE-160881-MS. http://dx.doi.org/ 10.2118/160881-MS.

Balhoff, M. T., Thompson, K. E. and Hjortsø, M. 2007. Coupling Pore-Scale Networks to Continuum-Scale Models of Porous Media. Comput. Geosci. 33 (3): 393-410. http://dx.doi.org/10.1016/j.cageo.2006.05.012.

Balay, S., Brown, J., Buschelman, K., et al. 2012. PETSc, http:// www.mcs.anl.gov/petsc.

Balhoff, M. T., Thomas, S. G. and Wheeler, M. F. 2008. Mortar Coupling and Upscaling of Pore-Scale Models. Computat. Geosci. 12 (1): 15-27. http://dx.doi.org/10.1007/s10596-007-9058-6.

Battiatoa, I., Tartakovskya, D. M., Tartakovsky, A. M., et al. 2011. Hybrid Models of Reactive Transport in Porous and Fractured Media. Adv. Water Resour. 34 (9): 1140-1150. http://dx.doi.org/10.1016/j.advwatres. 2011.01.012. 
Berendsen, H. J. C., Grigera, J. R. and Straatsma, T. P. 1987. The Missing Term in Effective Pair Potentials. J. Phys. Chem. 91 (24): 6269-6271. http://dx.doi.org/10.1021/j100308a038.

Bussi, G., Donadio, D. and Parrinello, M. 2007. Canonical Sampling Through Velocity Rescaling. J. Chem. Phys. 126: 014101. http:// dx.doi.org/10.1063/1.2408420.

Chu, J., Engquist, B., Prodanović, M., et al. 2012. A Multiscale Method Coupling Network and Continuum Models in Porous Media I: SteadyState Single Phase Flow. Multiscale Model Simul. 10 (2): 515-549. http://dx.doi.org/10.1137/110836201.

Chu, J., Engquist, B., Prodanović, M., et al. 2013. A Multiscale Method Coupling Network and Continuum Models in Porous Media II-Single- and Two-Phase Flows. Adv. Appl. Math. Model. Computat. Sci. 66: 161-185. http://dx.doi.org/10.1007/978-1-4614-5389-5_7.

Clark, M. M. 2009. Transport Modeling for Environmental Engineers and Scientists, second edition. New York City: John Wiley \& Sons, Inc.

Ennis-King, J., Preston, I. and Paterson, I. 2005. Onset of Convection in Anisotropic Porous Media Subject to a Rapid Change in Boundary Conditions. Phys. Fluids 17: 084107. http://dx.doi.org/10.1063/1.2033911.

E, W. and Engquist, B. 2003. The Heterognous Multiscale Methods. Comm. Math. Sci. 1 (1): 87-133. http://dx.doi.org/10.4310/CMS.2003. v1.n1.a8.

Firoozabadi, A. and Cheng, P. 2010. Prospects for Subsurface $\mathrm{CO}_{2}$ Sequestration. AIChE J. 56 (6): 1398-1405. http://dx.doi.org/10.1002/ aic.12287.

Firouzi, M. and Wilcox, J. 2012. Molecular Modeling of Carbon Dioxide Transport and Storage in Porous Carbon-Based Materials. Micropor. Mesopor. Mat. 158 (1 August): 195-203. http://dx.doi.org/10.1016/ j.micromeso.2012.02.045.

Firouzi, M. and Wilcox, J. 2013. Slippage and Viscosity Predictions in Carbon Micropores and Their Influence on $\mathrm{CO}_{2}$ and $\mathrm{CH}_{4}$ Transport. J. Chem. Phys. 138: 064705. http://dx.doi.org/10.1063/1.4790658.

Hess, B., Kutzner, C., van der Spoel, D., et al. 2008. GROMACS 4: Algorithms for Highly Efficient, Load-Balanced, and Scalable Molecular Simulation. J. Chem. Theory Comput. 4 (3): 435-447. http://dx.doi. org/10.1021/ct700301q

IPCC (Intergovernmental Panel on Climate Change). 2005. IPCC Special Report on Carbon Dioxide Capture and Storage. New York City: Cambridge University Press.

Jorgensen, W. L. and Tirado-Rives, J. 1988. The OPLS Potential Functions for Proteins, Energy Minimizations for Crystals of Cyclic Peptides and Crambin. J. Am. Chem. Soc. 110 (6): 1657-1666. http:// dx.doi.org/10.1021/ja00214a001.

Michael, K., Golab, A., Shulakova, V., et al. 2011. Geological Storage of $\mathrm{CO}_{2}$ in Saline Aquifers-A Review of the Experience from Existing Storage Operations. Int. J. Greenhouse Gas. Contr. 4 (4): 659-667. http://dx.doi.org/10.1016/j.ijggc.2009.12.011.

Mills, R. T., Sripathi, V., Mahinthakumar, G., et al. 2009. Experiences and Challenges Scaling PFLOTRAN, a PETSc-based Code for Subsurface Reactive Flow Simulations, Toward the Petascale on Cray XT Systems. Oral presentation given at the 2009 Cray User Group Meeting, Atlanta, Georgia, 4-7 May.

Nosé, S. and Klein, M. L. 1983. Constant Pressure Molecular Dynamics for Molecular Systems. Mol. Phys. 50 (5): 1055-1076. http:// dx.doi.org/10.1080/00268978300102851.

Parrinello, M. and Rahman, A. 1981. Polymorphic Transitions in Single Crystals: A New Molecular Dynamics Method. J. Appl. Phys. 52: 7182-7190. http://dx.doi.org/10.1063/1.328693.

Paschek, D. 2004. Temperature Dependence of the Hydrophobic Hydration and Interaction of Simple Solutes: An Examination of Five Popular Water Models. J. Chem. Phys. 120: 6674-6690. http://dx.doi.org/ $10.1063 / 1.1652015$.

Pau, G. S. H., Bell, J. B., Pruess, et al. 2010. High-Resolution Simulation and Characterization of Density-driven Flow in $\mathrm{CO}_{2}$ Storage in Saline Aquifers. Adv. Water Resour. 33 (4): 443-455. http://dx.doi.org/ 10.1016/j.advwatres.2010.01.009.

Pruess, K. and Zhang, K. 2008. Numerical Modeling Studies of The Dissolution-Diffusion-Convection Process During $\mathrm{CO}_{2}$ Storage in Saline Aquifers. Technical report, Material Sciences Division, Lawrence Berkeley National Laboratory, University of California, Berkeley, California (December 2008).
Sheng, Q. and Thompson, K. 2013. Dynamic Coupling of Pore-Scale and Reservoir-Scale Models for Multiphase Flow. Water Resour. Res. 49 (9): 5973-5988. http://dx.doi.org/10.1002/wrcr.20430.

Slim, A. C. and Ramakrishnan, T. 2010. Onset and Cessation of TimedDependent, Dissolution-Driven Convection in Porous Media. Phys. Fluids 22: 124103. http://dx.doi.org/10.1063/1.3528009.

van der Spoel, D., Lindahl, E., Hess, B., et al. 2010. Gromacs, Version 4.5.4 User Manual. http://www.gromacs.org.

Sun, T., Mehmani, Y. and Balhoff, M. 2012. Hybrid Multiscale Modeling through Direct Substitution of Pore-Scale Models into Near-Well Reservoir Simulators. Energ. Fuel. 26 (9): 5828-5836. http://dx.doi.org/ 10.1021/ef301003b.

Tartakovsky, A. M., Tartakovsky, D. M., Scheibe, T. D., et al. 2008. Hybrid Simulations of Reaction-Diffusion Systems in Porous Media. SIAM J. Sci. Comput. 30 (6): 2799-2816. http://dx.doi.org/10.1137/ 070691097.

Yan, M., Jordan, K. E., Kaushik, D., et al. 2012. Coupling a Basin Modeling and a Seismic Code Using MOAB. Procedia Comput. Sci. 9: 986-993. http://dx.doi.org/10.1016/j.procs.2012.04.105.

Zhang, Z. and Duan, Z. 2005a. Prediction of the PVT Properties of Water Over Wide Range of Temperatures and Pressures from Molecular Dynamics Simulation. Phys. Earth Planet In. 149 (3-4): 335-354. http:// dx.doi.org/10.1016/j.pepi.2004.11.003.

Zhang, Z. and Duan, Z. 2005b. An Optimized Molecular Potential for Carbon Dioxide. J. Chem. Phys. 122: 214507. http://dx.doi.org/10.1063/ 1.1924700 .

Kai Bao is a research scientist in the Department of Applied Mathematics at SINTEF ICT. Previously, he worked for more than 3 years as a post-doctoral fellow at King Abdullah University of Science and Technology (KAUST), Saudi Arabia. Bao's current research interests include reservoir simulation, parallel computing, $\mathrm{CO}_{2}$ sequestration, and enhanced oil recovery. $\mathrm{He}$ holds a bachelor's degree in thermal engineering from $\mathrm{Xi}^{\prime}$ an Jiaotong University, China, and a PhD degree in computer applied technology from Institute of Software, Chinese Academy of Sciences.

Mi Yan is a senior high-performance specialist at lonic Security. Previously she has worked as a high-performance specialist at IBM and as a senior software engineer at Linux Networx. Yan's interests include compiler, parallel algorithms, optimization, scientific computing, and data science. She has extensive experience in parallel applications including computational biology, medical imaging, seismic imaging, and molecular dynamics. Yan has coauthored more than 10 technical papers and holds six patents. She holds a PhD degree in computer engineering from the University of New Mexico.

Rebecca Allen is a post-doctoral researcher at SINTEF ICT. Her research interests include modeling flow and transport problems related to geological $\mathrm{CO}_{2}$ storage. Allen is an SPE member, and was an executive member for the SPE student chapter at King Abdullah University of Science and Technology, Saudi Arabia. She holds a PhD degree in earth science and engineering from King Abdullah University of Science and Technology.

Amgad Salama is an adjunct research scientist at the Reservoir Engineering Research Institute. Previously, he pursued his research at Kyushu University, Japan; Konkuk University, Korea; and King Abdullah University of Science and Technology. Salama's research interest is interdisciplinary, bridging the borders between several fields including petroleum, environmental, civil, mechanical, and nuclear engineering as well as earth science. His primary research interest is related to transport phenomena in porous media, particularly in subsurface reservoirs, spanning a wide range of length scales pertinent to the complex processes that take place in porous media. Salama holds bachelor's and master's degrees in mechanical engineering and PhD degree in environmental engineering from Carleton University, Canada.

Ligang Lu is currently with Shell International Exploration and Production, leading two research-and-development teams in seismic-data compression and data analytics for oil and gas exploration. Before joining Shell, Lu was a research staff member at IBM T.J. Watson Research Center. His current research 
interests include data analytics and machine learning, seismic-data compression, and high-performance computing for oil and gas industry applications. Lu has authored or coauthored more than 60 peer-reviewed technical papers and holds more than 40 issued or pending patents. He was a cochair of the Institute of Electrical and Electronics Engineers International Conference on Visual Communications and Image Processing 2008 and was chair of the IBM Research Signal Processing Professional Interest Community. Lu currently serves on the committee of 2016 Oil and Gas High Performance Computing Conference. He is a member of SPE. Lu holds a PhD degree in electrical engineering from Rensselaer Polytechnic Institute, where he was also awarded the Allan Dumont Prize for his doctoral work.

Kirk E. Jordan is an IBM distinguished dngineer in the data-centric systems organization, an IBM technical executive position in IBM's Research Division responsible for high-performance-computing direction and strategy. He has been with IBM for more than 20 years. Jordan oversees development of applications for IBM's advanced computing architectures, investigates and develops concepts for new areas of growth involving high-performance computing, and provides leadership in high-end data-centric computing and simulation in such areas as geoscience, computational fluid dynamics, systems biology, and high-end visualization. He is a member of the IBM Academy of Technology, a fellow of the Society for Industrial and Applied Mathematics, and a fellow of the American Association for Advancement of Science. He holds a PhD degree in applied mathematics from the University of Delaware.

Shuyu Sun is a founding faculty member of KAUST, and currently he serves as the codirector of KAUST's Consortium for Fluid Modeling and Seismic Imaging. He is an associate professor of earth sciences and engineering at KAUST, with a joint faculty appointment with the Applied Mathematics and Com- putational Science program. Sun also holds adjunct faculty appointments with Clemson University; Xi'an Jiao Tong University, China; and China University of Petroleum at Beijing. Before joining KAUST, he served as an assistant professor of mathematical sciences at Clemson University. Sun's research includes the modeling and simulation of flow and reactive transport in porous media and numerical analysis of relevant algorithms. In recent years, his research team at KAUST is investigating multiscale numerical algorithms for coupling Darcyscale, pore-scale, and molecular-scale models as applied to subsurface compositional flow with emphasis in modeling enhanced oil recovery and carbon sequestration. Sun has published more than 200 research papers and technical reports, which include more than 100 refereed journal articles. $\mathrm{He}$ is a member of a number of honor societies including Sigma Xi, Tau Beta Pi, Gamma Beta Phi, and Phi Kappa Phi. Sun achieved the certification under the SPE Petroleum Engineering Certification Program, and he is a licensed professional engineer in Texas. He has been a member of SPE since 2005. Sun is the current and founding faculty adviser of the SPE Student Chapter at KAUST, and in 2013 he received a certification from SPE recognizing him as "serving exceptionally well as Student Chapter Faculty Adviser" for the KAUST chapter. Sun holds a PhD degree in computational and applied mathematics from the University of Texas at Austin.

David Keyes is Director of the Extreme Computing Research Center at KAUST, where he was a founding dean. His primary interests are in scalable implicit solvers for nonlinear and linear algebraic systems. Keyes is a fellow of the Society for Industrial and Applied Mathematics and the American Mathematical Society, and has received the Association for Computing Machinery Gordon Bell Prize and the Institute of Electrical and Electronics Engineers Sidney Fernbach Award. He holds a bachelor's degree from Princeton University and a PhD degree from Harvard University. 